\title{
Nuclei micro-array FISH, a desirable alternative for MCL diagnosis
}

\author{
Hongjuan Chu $\cdot$ Xiqun Han $•$ Huiyong Jiang $\cdot$ Feng Li $•$ \\ Huiling Li • Tong Zhao
}

Received: 1 March 2010 /Accepted: 9 February 2011 / Published online: 23 February 2011

(C) The Author(s) 2011. This article is published with open access at Springerlink.com

\begin{abstract}
Mantle cell lymphoma (MCL) is a rare, specific lymphoma subtype. Though the morphologic and immunophenotypic features of MCL have been well described in recent literatures, it is still a diagnostic dilemma because of its frequent confusion with other small B cell lymphomas (SBCLs). In the present study, we primarily focus on establishing a sensitive and specific method for the diagnosis of MCL, which is efficient to distinguish this disease from other SBCLs. We carried out our investigation for MCL and other SBCLs (including SLL, FL, MZL, and MALT) on their feature of morphology, immunophenotype, and $\mathrm{t}(11 ; 14)(\mathrm{q} 13 ; \mathrm{q} 32)$ translocation analysis based on polymerase chain reaction (PCR) and interphase nuclei micro-array fluorescence in situ hybridization (FISH). The morphologic and immunologic analysis showed the positive rate of cyclin D1 was $76.47 \%$ in MCL, which was significantly higher than that in other SBCLs. The positive rate of $\mathrm{t}(11 ; 14)$ translocation was $25.81 \%$ and $35.48 \%$, respectively, tested by general and semi-nested PCR, while $93.10 \%$ positive rate was shown with low background and strong signals pattern when tested by Nuclei micro-array FISH. Our research shows that $\mathrm{t}(11 ; 14)$ translocation is a special and useful diagnostic marker for MCL, and
\end{abstract}

Xiqun Han and Hongjuan Chu contributed equally to this work.

H. Chu $\cdot$ H. Jiang $\cdot$ F. Li $\cdot$ T. Zhao $(\bowtie)$

Department of Pathology, Southern Hospital,

Southern Medical University,

Guangzhou 510515, Guangdong Province,

People's Republic of China

e-mail: zhaotongketizu@126.com

X. Han $\cdot$ H. Li $\cdot$ T. Zhao

Key Laboratory of Molecular Tumor Pathology of Guangdong

Province, Southern Medical University,

Guangzhou 510515, People's Republic of China detection of the marker by nuclei micro-array FISH is convenient and economic, especially more sensitive and specific than other methods for the diagnosis of MCL.

Keywords Mantle cell Lymphoma $\cdot t(11 ; 14)(q 13 q 32)$ translocation · Nuclei micro-array · Fluorescence in situ Hybridization (FISH) $\cdot$ IgH/CCND1 gene .

Immunohistochemistry

\section{Introduction}

Mantle cell lymphoma (MCL) is a distinctive disease entity, accounting for approximately $3 \%$ to $10 \%$ of non-Hodgkin's lymphomas. Unlike other SBCLs, MCLs generally manifest aggressive clinical course with a median survival of only 3 to 5 years, while many are refractory to conventional therapy [1]. Due to widely different therapeutic regimens and prognosis, the differential diagnosis of MCL to other SBCLs is critical. Three different architectural patterns of MCL exist, including mantle zone, nodular, and diffuse pattern [2], which may be confused in morphology with other small B cell lymphomas (SBCLs), making it difficult to diagnose based purely on morphology. Immunohistochemistry may be helpful in some cases [3], but falsenegative or false-positive results can also lead to confusion due to the variable immunophenotype and/or poor quality of the tissue that acquired. Therefore, morphology and immunophenotype alone are not sufficient enough to establish the definitive diagnosis of MCL. Recent studies concentrate on the specificity of molecular genetics $t(11 ; 14)$ (q13;q32), which is characteristic of MCL [4] and can be detected by cytogenetic techniques, including polymerase chain reaction (PCR) and fluorescence in situ hybridization (FISH). However, conventional cytogenetic techniques 
PCR are less useful because of the variability of breakpoints, which results in low sensitivity [5]. So, FISH is the most sensitive technique for demonstrating the chromosome translocation. In this study, we introduced a new FISH procedure to establish a sensitive and effective method for the diagnosis and differential diagnosis of MCL in clinical laboratories.

\section{Materials and methods}

One hundred and eighteen cases of MCL and other SBCLs diagnosed during the period 2002 to 2006, in the Pathology Department of the Affiliated Nanfang Hospital of Southern Medical University, were reviewed on the basis of morphologic and immunophenotyping studies. Lymphoma cases were diagnosed according to the criteria from the new World Health Organization and classified by two separate expert hematopathologists.

\section{Morphology}

For morphologic interpretation, $2-\mu \mathrm{m}$ sections taken from $10 \%$ neutral buffered formalin-fixed, paraffin-embedded tissue were routinely stained with H\&E. The slides with tissues of MCL were evaluated for the pattern of involvement (mantle zone, nodular, or diffuse), as previously described [6].

\section{Immunohistochemistry}

Ninety-one of the 118 cases were stained with a panel of antibodies including CD20, CD10, CD5, Bcl-2, CD23, and cyclin D1 (Dako), using standard technique as described in other literature [7] with slight refining. For cyclin D1 staining, antigen retrieval was performed with $0.1 \mathrm{mmol} / \mathrm{L}$ ethylenediaminetetraacetic acid (EDTA) and with $\mathrm{pH} 8.0$ for $13 \mathrm{~min}$ at $121^{\circ} \mathrm{C}$ in an autoclave, and before antigen retrieval, the slides were predigested in $0.4 \%$ pepsin. Primary antibodies were incubated in a humidified chamber overnight at $4^{\circ} \mathrm{C}$, and appropriate positive and negative controls were set throughout the study.

\section{Molecular biology}

\section{Detection of $t(11 ; 14)$ translocation by PCR}

In total, 73 cases were selected for PCR study. Genomic DNA was extracted from paraffin-embedded tissue sections by the standard method described previously [8]. Both common and semi-nested PCR were introduced to detect the $t(11 ; 14)$ translocation. PCR primers directed to detect breakpoints within the major translocation cluster (MTC) were selected and performed as previously described[9]. For common PCR, a definite and sharp single band about $150-350 \mathrm{bp}$ on $2 \%$ agarose gel is considered as positive, and for semi-nested PCR, a definite and clear single band about $180-250$ bp on $2 \%$ agarose gel is positive.

\section{Detection of $t(11 ; 14)$ translocation by FISH}

Nuclei were extracted according to the method described previously [10] with slight modification. Instead of room temperature, water bath heating was applied for dewaxing. When the nuclei suspensions were ready, a micro-array map with a grid of $0.3 \mathrm{~cm}$ in diameter was made using a computer, a poly-L-lysine-coated slide was put on the micro-array map and the nuclei suspensions were dropped in accordance with the grid in the map, and then the slide were stored at room temperature for drying and eventually stored at $4^{\circ} \mathrm{C}$ for the following experiments.

FISH for detection of $\mathrm{t}(11 ; 14)$ translocation was performed on nuclei micro-array slides using commercially available LSI IgH/CCND1 dual-color, dual-fusion translocation probe, which was a mixture of the LSI IgH probe labeled with Spectrum Green and the LSI CCND1 probe labeled with Spectrum Orange (Vysis). The nuclei microarray slides were pretreated as described previously [11]. Hybridization was processed by diluting probe in hybridization buffer (Vysis, Downers Grove, IL, USA) and incubation at $82^{\circ} \mathrm{C}$ for $10 \mathrm{~min}$ and at $37 \mathrm{C}$ overnight. After hybridization, the samples were strictly washed as described by manufacturer and then counterstained with DAPI (Vysis). Finally, the specimen slides were evaluated under fluorescent microscope. At least 100-200 non-overlapping, intact nuclei were scored, and nuclei were counted only when they contained at least one bright-green signal and one bright-orange signal to avoid insufficient hybridization efficiency. Appropriate positive and negative controls were included. The cut-off level for the diagnosis of fusion was obtained from the results of the hybridization with chronic tonsillitis (mean \pm 3 standard deviation).

\section{Statistics}

The data were collected and statistically analyzed using the Statistical Package SPSS, VERSION 13.0 software program. $P$ values of $<0.05$ was considered statistically significant.

\section{Results}

Morphology

One hundred and eighteen cases consisting of $39 \mathrm{MCL}$ (here, we choose only SBCLs as the research subject, 
Table 1 Clinical features of small B cell lymphomas

\begin{tabular}{llllll}
\hline Characteristics & MCL $(N=39)$ & B-SLL $(N=19)$ & FL $(N=24)$ & MALT $(N=25)$ & MZL $(N=11)$ \\
\hline Sex (male/female) & $31 / 8$ & $11 / 8$ & $15 / 9$ & $15 / 10$ & $9 / 2$ \\
Age (range (mean)) & $23-72(58.36)$ & $16-73(50.58)$ & $26-79(52.38)$ & $13-81(51.48)$ & $36-76(58.82)$ \\
Age $\geqq 60$ & 21 & 11 & 9 & 8 & 7 \\
Localization (nodal/extranodal) & $26 / 13$ & $7 / 12$ & $19 / 5$ & $1 / 24$ & $3 / 8$ \\
\hline
\end{tabular}

$M C L$ mantle cell lymphoma, FL follicular lymphoma, B-SLL B cell small lymphocytic lymphoma, MZL marginal zone lymphoma, MALT mucosa-associated lymphatic tissue

among which, the proportion of MCL is much higher than that in general lymphomas), 19 B cell small lymphocytic lymphoma (B-SLL), 24 follicular lymphoma (FL), 11 marginal zone lymphoma (MZL), and 25 mucosaassociated lymphatic tissue (MALT) were confirmed with morphologic analysis. Patients were mostly in middle age or elderly adults with a male predominance. The main initial characteristics of the 118 patients were detailed in Table 1. For patients with MCL, the patterns of involvement included mantle zone (11 cases), nodular (12 cases), and diffuse (16 cases). Cases of MCL showed monotonous population of cells with small slightly cleaved nuclei, which showed morphologic features that overlap with other SBCLs. (Fig. 1).

Immunohistochemistry

Only 91 of the 118 cases were performed with immunohistochemical studies due to the lack of sufficient materials (including five MCL cases). Among the $34 \mathrm{MCL}$ cases that were assessed for immunohistochemistry, 26 (76.47\%) were reactive to cyclin D1 with moderate to strong intensity staining, the positive rate was significantly higher than in other SBCLs whereas some other SBCLs also showed cyclin D1 expression with weak/negative intensity. Our study showed that CD5 expression in MCL and B-SLL was low and only 19 of 34 cases (55.88\%) of MCL and three of ten of B-SLL were positive for CD5, lower than in other reports. Six of ten cases (60\%) of B-SLL that were performed on immunohistochemistry showed CD23 expression, which could also be detected in some MALT cases, but the remaining cases were negative for the CD23 antigen. CD10 was mainly detected in FL except for one MZL. None of the other cases was positive for CD10 expression. Bcl-2 was variably expressed in different types of the above lymphomas (Fig. 2).

\section{Molecular biology}

Some cases were not fit for PCR analysis to be performed on because of poor DNA quality. Of the 73 cases that were tested for $\mathrm{t}(11 ; 14)$ translocation by PCR, only the cases of
Fig. 1 Histological features of MCL and other similar small B cell lymphomas. MCL, MALT, B-SLL, and FL were shown to contain a monotonous population of cells with similar slightly cleaved nuclei $(\mathrm{H} \& \mathrm{E} \times 400)$

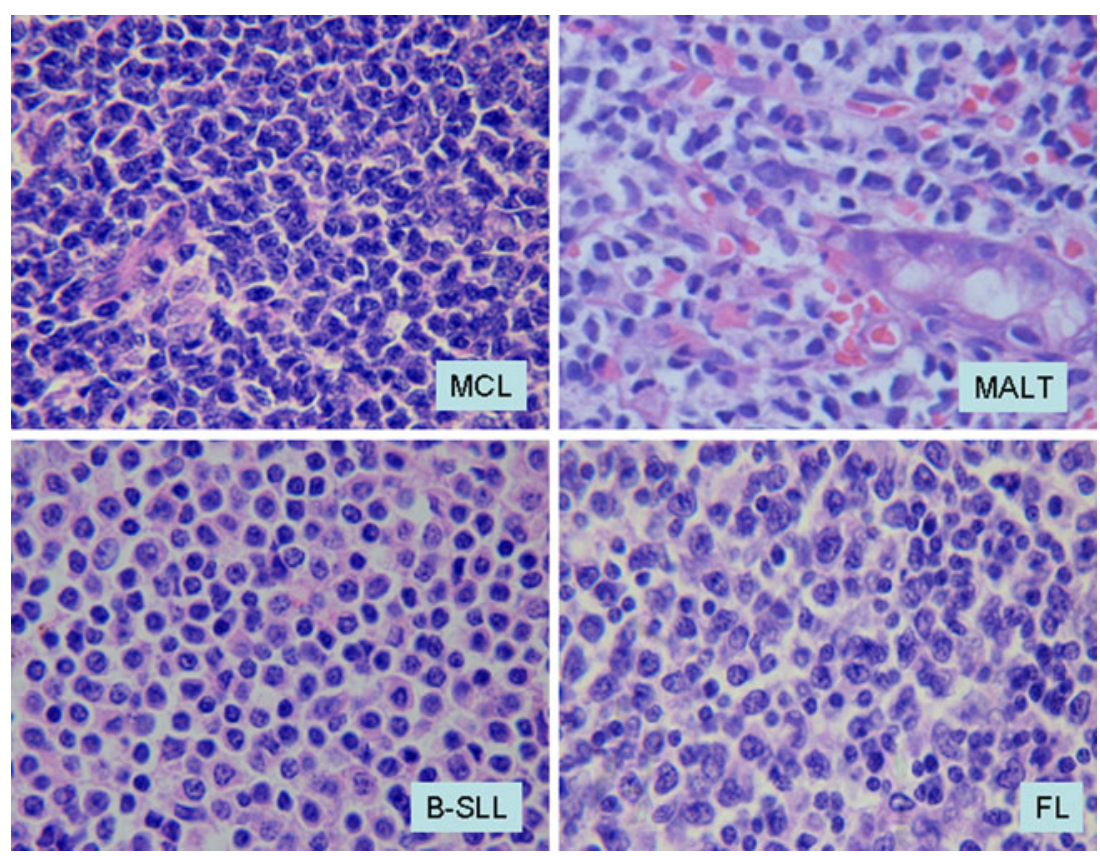



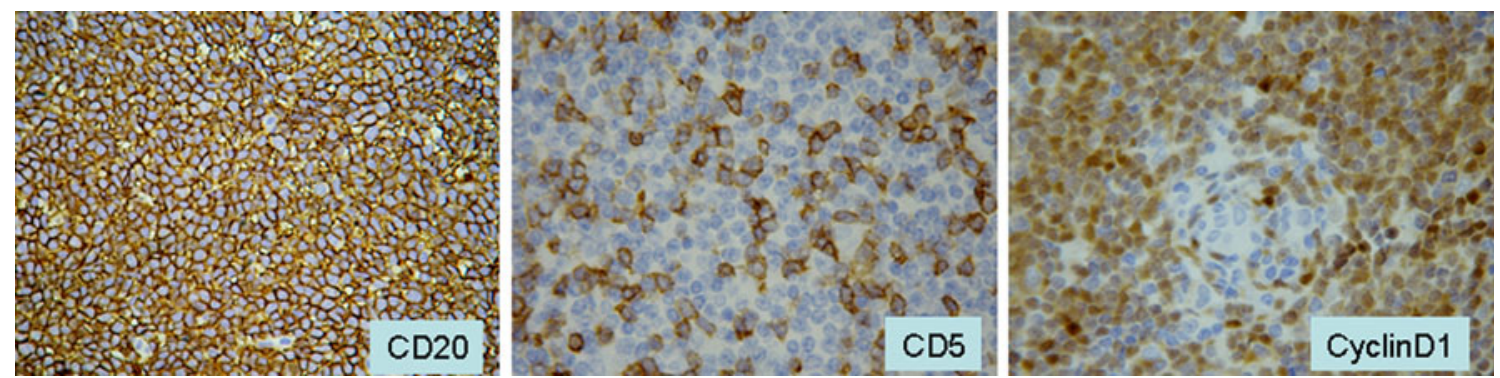

Fig. 2 Immunohistochemical staining for cases of MCL (magnification, $\times 400$ )

MCL were detected to be $\mathrm{t}(11 ; 14)$ translocation positive, while the others were negative. Eight of 31 (25.81\%) MCL were positive for $\mathrm{t}(11 ; 14)$ translocation by common PCR, while 13 of 31 (35.48\%) MCL were positively detected by semi-nested PCR (Fig. 3). Of the eight positive cases showing $\mathrm{t}(11 ; 14)$ translocation by common PCR, seven were also positive for $\mathrm{t}(11 ; 14)$ translocation in semi-nested PCR studies while the remaining one was negative. Though semi-nested PCR had greater sensitivity, there was no statistically significant difference between the two methods, $p=0.409(p>0.05)$.

Seven of the 73 cases including two MCL, four B-SLL, and one MALT failed to have been extracted adequate nuclei to make nuclei micro-array due to inadequate tissue. The remaining 66 cases showed that the quality of nuclei extracted with water bath heating instead of on common temperature for dewaxing was higher with few foreign materials, and the nuclei micro-array made by pointing nuclei on the slides directly was qualified with clear background and steady nuclei. Among the 66 cases studied with FISH, two cases with chronic tonsillitis failed to produce adequate signal for counting. FISH results in normal lymphocyte nuclei showed the expected two orange and two green probe signals, while results in tumor cell nuclei showed one orange probe (CCND1), one green probe $(\operatorname{IgH})$, and one or two yellow fusion $(\operatorname{IgH} / \mathrm{CCND})$ probe signals patterns (Fig. 4). The $\mathrm{t}(11 ; 14)$ translocation were detected in 27 of $29(93.10 \%)$ cases of MCL with low background and strong signal patterns, and the fusion signals were detected in $40-85 \%$ of the counted nuclei. Specifically, three cases of B-SLL were also detected to be $t$ $(11 ; 14)$ translocation positive, of which, one was weak positive, one was weak/negative, and one was negative for cyclin D1expression in prior immunohistochemical studies. Among the remaining cases that were evaluated, no cases harbored $\mathrm{t}(11 ; 14)$ translocation. Notably, there were two cases of MCL showing CCND1 gene amplification with more than three orange signals in one nucleus, and 50-80\% of the counted nuclei showed these amplification signals.

The results of FISH, PCR, and Immunophenotyping of the 34 cases of MCL are detailed in Table 2.

\section{Discussion}

MCL is now recognized as a distinct clinicopathologic entity. It is a rare type of lymphoma comprising between $5 \%$ and $10 \%$ of all non-Hodgkin's lymphomas. It is very important to recognize this rare lymphoma because of its poor overall survival rate and limited response to conventional chemotherapy in most cases. Several morphologic variants have been described, including mantle zone, nodular, and diffuse growth patterns. Some researches showed that patients with mantle zone pattern apparently had a more indolent outcome, while patients with nonnodal pattern seemed to have a better prognosis when compared with patients with predominantly nodular pattern [12]. In our study, the patterns of involvement included mantle zone (11 cases), nodular (12 cases), and diffuse (16 cases), but we failed to proceed with the analysis due to semi-complete clinical data. Our study also showed cases of MCL having morphologic overlaps with other SBCLs, making the primary diagnosis and subclassification based on morphology alone a diagnostical challenge. Thus, the
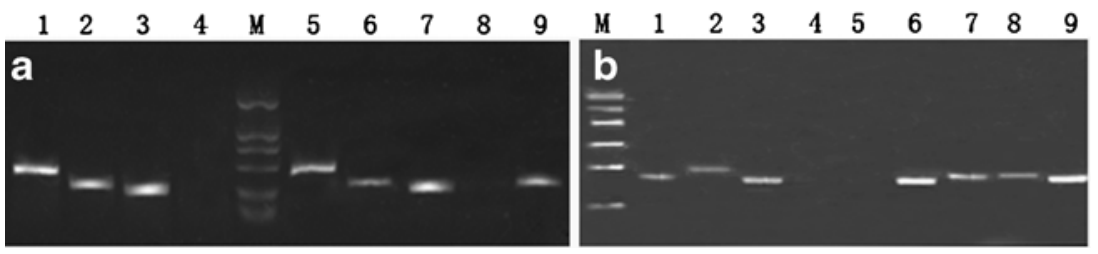

Fig. 3 Detection of $\mathrm{t}(11 ; 14)$ translocation by PCR. $M$ DNA marker, 100 bp. a Common PCR: lanes 1-3 and 5-9 were cases of MCL; lane 4 was negative control; lanes $1-3,5-7$, and 9 show a sharp single- positive band about 150-350 bp. b Semi-nested PCR: lanes 1-3 and 5-9 were cases of MCL; lane 4 was negative control; lanes 1-3 and 6-9 show a clear single band about $180-250 \mathrm{bp}$ 
Fig. 4 a Chronic tonsillitis case showing normal signal patterns with two green and two orange signals, $\times 630$. b Case of MCL showing abnormal signal patterns with colocalization of green and orange signals, $\times 630$
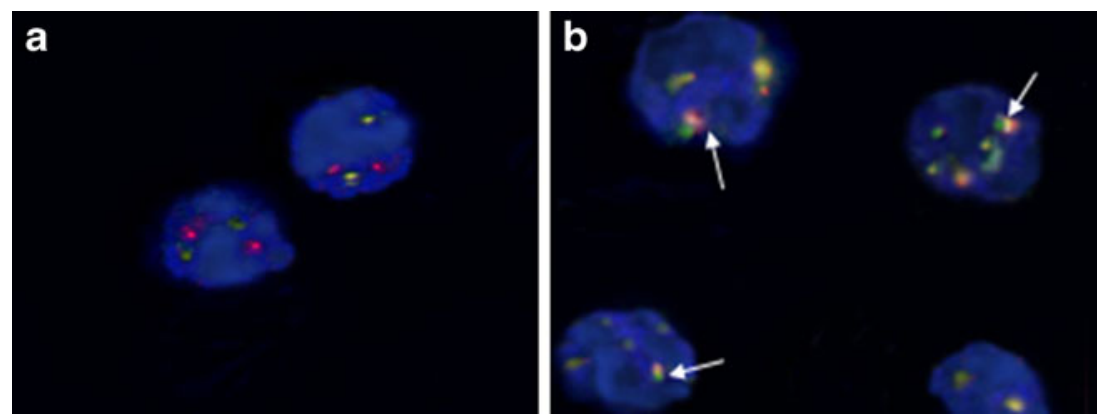

Table 2 Results of FISH, PCR, and Immunophenotyping study in MCL

\begin{tabular}{|c|c|c|c|c|}
\hline \multirow[t]{2}{*}{ Patient no. } & \multirow{2}{*}{$\begin{array}{l}\text { FISH t(11;14) } \\
(\mathrm{q} 13 ; \mathrm{q} 32)\end{array}$} & \multicolumn{2}{|c|}{ PCR t(11;14)(q13;q32) } & \multirow{2}{*}{$\begin{array}{l}\text { Cyclin D1 } \\
\text { staining }\end{array}$} \\
\hline & & Common & Semi-nested & \\
\hline 1 & Positive & Negative & Negative & Positive \\
\hline 2 & Positive & Negative & Negative & Positive \\
\hline 3 & Positive & Negative & Negative & Negative \\
\hline 4 & Failed & Negative & Negative & Positive \\
\hline 5 & Positive & Positive & Positive & Positive \\
\hline 6 & Not do & Not do & Not do & Negative \\
\hline 7 & Not do & Not do & Not do & Positive \\
\hline 8 & Positive & Negative & Positive & Positive \\
\hline 9 & Positive & Negative & Negative & Positive \\
\hline 10 & Positive & Negative & Positive & Positive \\
\hline 11 & Positive & Negative & Positive & Positive \\
\hline 12 & Positive & Negative & Negative & Positive \\
\hline 13 & Positive & Negative & Negative & Negative \\
\hline 14 & Positive & Negative & Negative & Positive \\
\hline 15 & Positive & Positive & Positive & Positive \\
\hline 16 & Positive & Negative & Negative & Positive \\
\hline 17 & Not do & Not do & Not do & Negative \\
\hline 18 & Positive & Positive & Positive & Positive \\
\hline 19 & Positive & Negative & Positive & Positive \\
\hline 20 & Positive & Positive & Positive & Positive \\
\hline 21 & Failed & Negative & Negative & Negative \\
\hline 22 & Positive & Positive & Positive & Positive \\
\hline 23 & Positive & Negative & Positive & Positive \\
\hline 24 & Positive & Negative & Negative & Positive \\
\hline 25 & Positive & Positive & Positive & Positive \\
\hline 26 & Positive & Negative & Negative & Positive \\
\hline 27 & Positive & Negative & Negative & Positive \\
\hline 28 & Positive & Negative & Negative & Positive \\
\hline 29 & Positive & Positive & Negative & Positive \\
\hline 30 & Positive & Negative & Negative & Positive \\
\hline 31 & Positive & Positive & Positive & Positive \\
\hline 32 & Negative & Negative & Negative & Negative \\
\hline 33 & Negative & Negative & Negative & Negative \\
\hline 34 & Positive & Negative & Positive & Negative \\
\hline
\end{tabular}

FISH fluorescence in situ hybridization, PCR polymerase chain reaction availability of immunohistochemical markers is mandatory for the diagnosis. Positive cyclin D1 staining is a hallmark of MCL, and coexpression of cyclin D1 and CD5 can distinguish MCL from other SBCLs [13]. However, a minor proportion of the cases may fail to express cyclin D1 [14]. Clinically similar to the cases with cyclin D1 expression, the negative cases expressing cyclin D2 or cyclin D3 while maintaining the gene expression profile are common to the cyclin D1-positive MCL. In this situation, the absence of $\mathrm{CD} 23$ distinguishes MCL from B-SLL, and the presence of CD5 distinguishes MCL from FL and MZL [15]. However, expression of CD23 can be observed in a subset of MCL, which leads to diagnostic confusion [16]. Moreover, the results of immunophenotype are variable and sometimes may be false negative, mainly due to the quality of the material. In our study, though the detection rate of cyclin D1 can be improved by performing antigen retrieval by boiling in EDTA and predigesting in $0.4 \%$ pepsin before antigen retrieval, the positive rate still remains $76.47 \%$, only a little higher than that in other report [5]. The negative results usually made us confused, and it was hard to determine whether they were true or false negative due to various staining with different fixation. Obviously, immunohistochemistry alone is not sufficient to establish a definitive diagnosis, and molecular analysis may be crucial for the diagnosis.

The hall marker of MCL is $t(11 ; 14)$ translocation, which is virtually present in all MCL, rarely involved with other lymphoma types [17]. Some researches used different methods to detect this translocation [18], but each of the methods had some shortages. Conventional cytogenetics, which requires more time and fresh tissue, may not be readily available to all practitioners. Archival materials in our study are not fit for those traditional methods. PCR is an efficient method with fast speed but sometimes with relatively low specificity and sensitivity. In the present study, both common and semi-nested PCR were performed to detect $\mathrm{t}(11 ; 14)$ translocation, the positive rate were $25.81 \%$ and $35.48 \%$, respectively, lower than the $50.00 \%$ that was reported in other literature [19]. This low sensitivity may be due to the specific chromosomal translocation, in which the actual breakpoints scattered 
over a large area spanning more than $100 \mathrm{~kb}$ on $11 \mathrm{q} 13$. Although the great majority of these breakpoints are located within a segment of about $100 \mathrm{bp}$ located around the MTC, there are still some cases with breakpoints out of the MTC [20]. Thus, PCR analysis may give false-negative results.

FISH with great value in cytogenetic studies is applied in many researches. It shows highly specific and sensitive, but the expensive probes make it unpractical to routine application. In this study, we made an ideal result by performing FISH on nuclei micro-array, which was established and reported by our research team in detection of $t$ $(14 ; 18)$ in FL. By performing FISH on nuclei micro-array slides, many cases could be finished simultaneously on a single slide with high comparability and little variance, making it more simple, rapid and inexpensive to be carried out. With some improvement, such as extracting nuclei by water bath heating for dewaxing, we obtained intact nuclei with few foreign materials. Meanwhile, by pointing nuclei on the slides directly to substitute the cell micro-array molds that were reported in our previous study [10], we made perfect nuclei micro-arrays with clear background and steady nuclei more easily and simply. Using this new method, we detected $\mathrm{t}(11 ; 14)$ translocation in $27 / 29$ of MCL with low background and strong signal patterns. The positive rate was $93.10 \%$, which was significantly higher than that of immunohistochemistry or PCR analysis. In fact, the two negative cases were abnormal and not suitable for this type of situation. Some cases showed typical features of MCL in morphology analysis, while the immunophenotyping results were confusing. They were positive for CD5, but cyclin $\mathrm{D} 1$ and $\mathrm{CD} 23$ were both negative. For some cyclin D1-negative cases of MCL reported in other study [21], they both were classified to MCL based on morphology. However, $\mathrm{t}(11 ; 14)$ translocation was not detected in these cases by nuclei micro-array FISH, so genuine MCL or not of these cases were questioned. Therefore, nuclei microarray FISH is the most sensitive technique when compared with immunohistochemistry and PCR. Specifically, the $t$ $(11 ; 14)$ translocation has been described in other lymphoid tumors, including hairy cell leukemia and multiple myeloma. In our present study, three cases of B-SLL were also detected $\mathrm{t}(11 ; 14)$ translocation, of which, one was weak positive, one was weak/negative and one was negative for cyclin D1 expression and all were weak positive for CD23 expression in prior immunohistochemical studies. Although there were a few patients with B-SLL/CLL have been reported to have $\mathrm{t}(11 ; 14)$ translocation [22], it was unclear whether the B-SLL/CLL harboring $\mathrm{t}(11 ; 14)$ translocation represent genuine CLL or MCL. Moreover, expression of CD23 can be observed in a subset of MCL [16], so, the three B-SLL with $\mathrm{t}(11 ; 14)$ translocation positive might be $\mathrm{MCL}$ indeed. Interestingly, we found two cases with MCL showed CCND1 gene amplification with more than three orange signals in one nucleus. The CCND1 gene encodes for cyclin D1, which has been shown to be expressed even in cases without $t(11 ; 14)$ translocation. Therefore, it has been suggested that CCND1could also be activated by amplification [23], though this requires more studies.

In conclusion, the diagnosis of MCL is often a dilemma and requires a combination of morphologic, immunohistochemical, and genetic studies. Our study shows that nuclei micro-array FISH is convenient and economic, suitiable for routine detection of MCL $t(11 ; 14)$ translocation in clinical laboratory, making it a promising and desirable alternative for MCL diagnosis.

Acknowledgments We gratefully thank Prof. Meigang Zhu for revising the manuscript, Dr. Henry Li and technician Xiangzhao Li for technical assistance. This study is supported by the Science and Technology Project of Guangzhou (no. 2002Z3-E4016) and Science and Technology Project of Guangdong (no. B30101).

Open Access This article is distributed under the terms of the Creative Commons Attribution Noncommercial License which permits any noncommercial use, distribution, and reproduction in any medium, provided the original author(s) and source are credited.

\section{References}

1. Jaffe ES, Harris NL, Diebold J et al (1999) World Health Organization classification of neoplastic diseases of the hematopoietic and lymphoid tissues. A progress report. Am J Clin Pathol 111(1 Suppl 1):8-12

2. Khosravi SP, Del CR, Perez MG (2007) Mantle cell lymphoma. Med Interna 24(3):142-145

3. Thieblemont C, Nasser V, Felman P et al (2004) Small lymphocytic lymphoma, marginal zone B-cell lymphoma, and mantle cell lymphoma exhibit distinct gene-expression profiles allowing molecular diagnosis. Blood 103(7):2727-2737

4. Parry-jones N, Matutes E, Morilla R et al (2007) Cytogenetic abnormalities additional to $t(11 ; 14)$ correlate with clinical features in leukaemic presentation of mantle cell lymphoma, and may influence prognosis: a study of 60 cases by FISH. Br J Haematol 137(2):117-124

5. Belaud-rotureau MA, Parrens M, Dubus P et al (2002) A comparative analysis of FISH, RT-PCR, PCR, and immunohistochemistry for the diagnosis of mantle cell lympho-mas. Mod Pathol 15(5):517-525

6. Weisenburger DD, Vose JM, Greiner TC et al (2000) Mantle cell lymphoma. A clinicopathologic study of 68 cases from the Nebraska Lymphoma Study Group. Am J Hematol 64(3):190-196

7. De BC, Schuuring E, Dreef E et al (1995) Cyclin D1 protein analysis in the diagnosis of mantle cell lymphoma. Blood 86 (7):2715-2723

8. Theriault C, Galoin S, Valmary S et al (2000) PCR analysis of immunoglobulin heavy chain (IgH) and TcR-gamma chain gene rearrangements in the diagnosis of lympho -proliferative disorders: results of a study of 525 cases. Mod Pathol 13(12):1269-1279

9. Van DJ, Langerak AW, Bruggemann M et al (2003) Design and standardization of PCR primers and protocols for detection of clonal immunoglobulin and T-cell rece -ptor gene recombinations in suspect lymphoproliferations: report of the BIOMED-2 Concerted Action BMH4-CT98-3936. Leukemia 17(12):2257-2317 
10. Paternoster SF, Brockman SR, McClure RF et al (1967) A new method to extract nuclei from paraffin-embedded tissue to study lymphomas using interphase fluorescence in situ hybridization. Am J Pathol 160(6):1967-1972

11. Hui-yong Jiang, San-quan Zhang, Tong Zhao (2006) A new method to make nuclei or cell microarrays. Diagn Mol Pathol 15 (2):109-114

12. Rubio-Moscardo F, Climent J, Siebert R et al (2005) Mantle-cell lymphoma genotypes identified with $\mathrm{CGH}$ to BAC microarrays define a leukemic subgroup of disease and predict patient outcome. Blood 105:4445-4454

13. Bertoni F, Ponzoni M (2007) The cellular origin of mantle cell lymphoma. Int J Biochem Cell Biol 39(10):1747-1753

14. Yatabe Y, Suzuki R, Tobinai K et al (2000) Significance of cyclin D1 overexpression for the diagnosis of mantle cell lymphoma: a clinicopathologic comparison of cyclin D1-positive MCL and cyclin D1-negative MCL-like B-cell lymphoma. Blood 95 (7):2253-2261

15. Harris NL, Jaffe ES, Stein H et al (1994) A revised EuropeanAmerican classification of lymphoid neoplasms: a propasal from the International Lymphoma Study Group. Blood 84:1361-1392

16. Gong JZ, Lagoo AS, Peters D et al (2002) Value of CD23 determination by flow cytometry in differentiating mantle cell lymphoma from chronic lymphocytic leukemia/small lymphocytic lymphoma. Am J Clin Pathol 116:893-897
17. Welzel N, Le T, Marculescu R et al (2001) Templated nucleotide addition and immuno-globulin JH-gene utilization in $t(11 ; 14)$ junctions: implications for the mechanism of translocation and the origin of mantle cell lymphoma. Cancer Res 61(4):1629-1636

18. Quintanilla-Martinez L, Slotta-Huspenina J, Koch I et al (2009) Differential diagnosis of cyclin D2+ mantle cell lymphoma (MCL) based on fluorescence in situ hybridization (FISH) and quantitative real-time-PCR. Haematologica 94(11):1595-8

19. Luthra R, Hai S, Pugh WC (1995) Polymerase chain reaction detection of the $\mathrm{t}(11 ; 14)$ translocation involving the bcl-1 major translocation cluster in mantle cell lymphoma. Diagn Mol Pathol 4 (1):4-7

20. Degan M, Doliana R, Gloghini A et al (2002) A novel bcl-1/JH breakpoint from a patient affected by mantle cell lymphoma extends the major translocation cluster. J Pathol 197(2):256-263

21. De Ageli C, Domenica G, Cuneo A et al (2000) BCL-1 rearrangements and p53 mutations in atypical chronic lymphocytic leukemia with $\mathrm{t}(11 ; 14)(\mathrm{q} 13 ; 32)$. Haematologica 85:913-921

22. Ana Mozos, Crlstlna Royo, Elena Hartmann et al (2009) SOX11 expression is highly specific for mantle cell lymphoma and identifies the cyclinD1-negative subtype. Haematologica 94(11):1555-1562

23. Monni O, Joensuu H, Franssila K, Klefstrom J, Alitalo K, Knuutila S (1997) BCL2 overexpression associated with chromosomal amplification in diffuse large B-cell lymphoma. Blood 90:1168-1174 